\title{
Universiteit
}

Leiden

The Netherlands

\section{Sunitinib-induced hypertension in CYP3A4 rs4646437 A-allele carriers with metastatic renal cell carcinoma}

Diekstra, M.; Belaustegui, A.; Swen, J.J.; , E. boven; Castellano, D.; Gelderblom, H.; ... ; Guchelaar, H.J.

\section{Citation}

Diekstra, M., Belaustegui, A., Swen, J. J., Castellano, D., Gelderblom, H., Mathijssen, R. H., ... Guchelaar, H. J. (2017). Sunitinib-induced hypertension in CYP3A4 rs4646437 A-allele carriers with metastatic renal cell carcinoma. Pharmacogenomics Journal, 17(1), 42-46. doi:10.1038/tpj.2015.100

Version: $\quad$ Not Applicable (or Unknown)

License: $\quad$ Leiden University Non-exclusive license

Downloaded from: https://hdl.handle.net/1887/114726

Note: To cite this publication please use the final published version (if applicable). 


\title{
ORIGINAL ARTICLE Sunitinib-induced hypertension in CYP3A4 rs4646437 A-allele carriers with metastatic renal cell carcinoma
}

\author{
MH Diekstra ${ }^{1,2}$, A Belaustegui $^{3}$, JJ Swen ${ }^{1,2}$, E Boven $^{2,4}$, D Castellano ${ }^{5,6}$, H Gelderblom $^{2,7}$, RH Mathijssen ${ }^{2,8}$, J García-Donas ${ }^{6,9}$, \\ C Rodríguez-Antona ${ }^{10,11}$, BI Rini ${ }^{12}$ and H-J Guchelaar ${ }^{1,2}$
}

The single nucleotide polymorphism (SNP) rs4646437G > A in CYP3A4 was suggested to be related to sunitinib toxicity. Our objective was to perform an in-depth investigation of the association between this SNP and sunitinib toxicity and efficacy using a large cohort of metastatic renal cell carcinoma (mRCC) patients. We collected DNA and clinical information of mRCC patients treated with sunitinib. SNP rs4646437 in CYP3A4 was tested for associations with toxicity using logistic regression. Cox regression modeling was used for association analysis of rs4646437 with progression-free survival (PFS) and overall survival (OS). In a total of 287 patients, the A-allele of CYP3A4 rs4646437 was associated with an increased risk for hypertension (odds ratio $=2.4,95 \%$ confidence interval: $1.1-5.2, P=0.021$ ) and showed no significant association with PFS or OS. In conclusion, hypertension is more likely to occur in A-allele carriers of the CYP3A4 rs4646437 variant in our cohort of mRCC patients treated with sunitinib.

The Pharmacogenomics Journal (2017) 17, 42-46; doi:10.1038/tpj.2015.100; published online 26 January 2016

\section{INTRODUCTION}

Sunitinib, a tyrosine kinase inhibitor, is widely prescribed for treatment of metastatic renal cell carcinoma (mRCC). ${ }^{1}$ The large inter-individual variability in sunitinib response makes it difficult to predict treatment outcome in individual patients. Sunitinibinduced adverse events lead to dose reductions or treatment discontinuation with subsequent loss of treatment efficacy. This large variability can be explained not only by patient characteristics (for example drug-drug interactions, treatment adherence and environmental factors) but also by the genetic profile of patients. Studies using the candidate gene approach have identified single nucleotide polymorphisms (SNPs) to be associated with the efficacy and risk of toxicity on sunitinib treatment. $^{1-5}$

Sunitinib is metabolized by CYP3A4 to its active metabolite SU12662. Urun et al. ${ }^{6}$ have observed that A-allele carriers of CYP3A4 rs4646437G $>$ A showed a lower risk of grades 3-4 toxicity (odds ratio $(\mathrm{OR})=0.27,95 \%$ confidence interval $(\mathrm{Cl})$ : $0.08-0.88$, $P=0.03)$ compared with wild-type (WT) GG in a cohort of 159 European $\mathrm{mRCC}$ patients treated with sunitinib. In a small number of reports on other types of drugs that are metabolized by CYP3A4, the variant A-allele of rs4646437 was associated with either an increased or decreased activity of CYP3A4. ${ }^{7-9}$ He et al. ${ }^{7}$ tested 22 SNPs in genes encoding CYP3A4, CYP3A5 and CYP2C19 for associations with voriconazole pharmacokinetics $(P K)$ in a sample of 158 patients. The presence of A-allele of rs4646437 was associated with high plasma voriconazole concentrations of $>4 \mathrm{mg} \mathrm{I}^{-1}(\mathrm{OR}=2.8,95 \% \mathrm{Cl}: 1.09-7.38, P=0.03)$. The authors note that, although rs4646437 is located in the intron
(99767460G $>A$ ), it could alter the splicing of primary transcripts or gene expression of CYP3A4. In another cohort of 240 Chinese renal transplant recipients receiving tacrolimus, Li et al. ${ }^{8}$ assessed 17 SNPs in CYP3A5, CYP3A4, COMT, IL-10 and POR for association with tacrolimus PK. For rs4646437, a significant association was observed with dose-adjusted tacrolimus blood concentration $\left(C_{0} / D\right)$ showing lower ratios for the variant A-allele carriers. ${ }^{8}$ Crettol et al. ${ }^{9}$ studied 73 patients receiving cyclosporine and reported that A-allele carriers of rs4646437 needed a higher dose of cyclosporine.

A potential explanation for the conflicting results of rs4646437 variant on CYP3A4 expression and metabolism may be related to gender. Schirmer et al. ${ }^{10}$ reported that rs4646437 is a sex-dependent genetic marker with a higher CYP3A4 expression and activity for female A-allele carriers compared with male A-allele carriers. Therefore, it was suggested to take gender into account in the genetic analyses of CYP3A4. ${ }^{10}$

The aim of the current study was to establish whether there is a relationship between rs4646437 in CYP3A4 and sunitinib treatment outcome in a large cohort of clear cell mRCC patients.

\section{MATERIALS AND METHODS}

Study population

This exploratory post-hoc analysis is one of the largest pharmacogenetic studies in mRCC patients. In this large observational study, DNA samples together with clinical information were collected from previous pharmacogenetic studies on sunitinib-treated clear cell mRCC patients enrolled between 2004 and 2010 (Figure 1). The SUTOX consortium is a Dutch

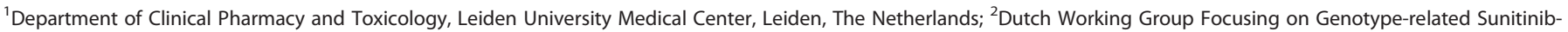

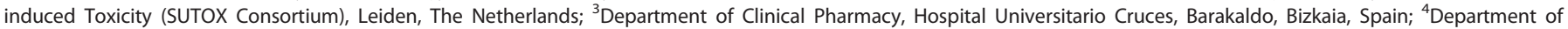

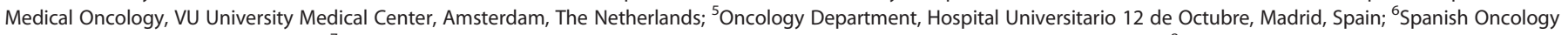

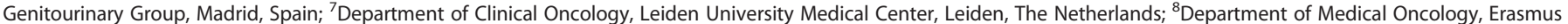

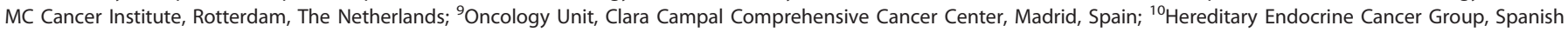

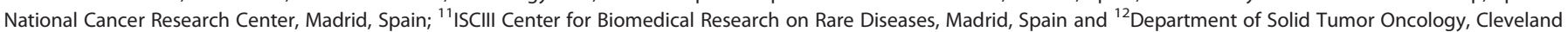

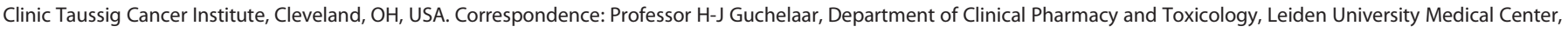
Albinusdreef 2, 2300RC Leiden, P.O. Box 9600, Post Zone L0-P, The Netherlands.

E-mail: H.J.Guchelaar@lumc.nl

Received 8 July 2015; revised 27 August 2015; accepted 16 October 2015; published online 26 January 2016
} 


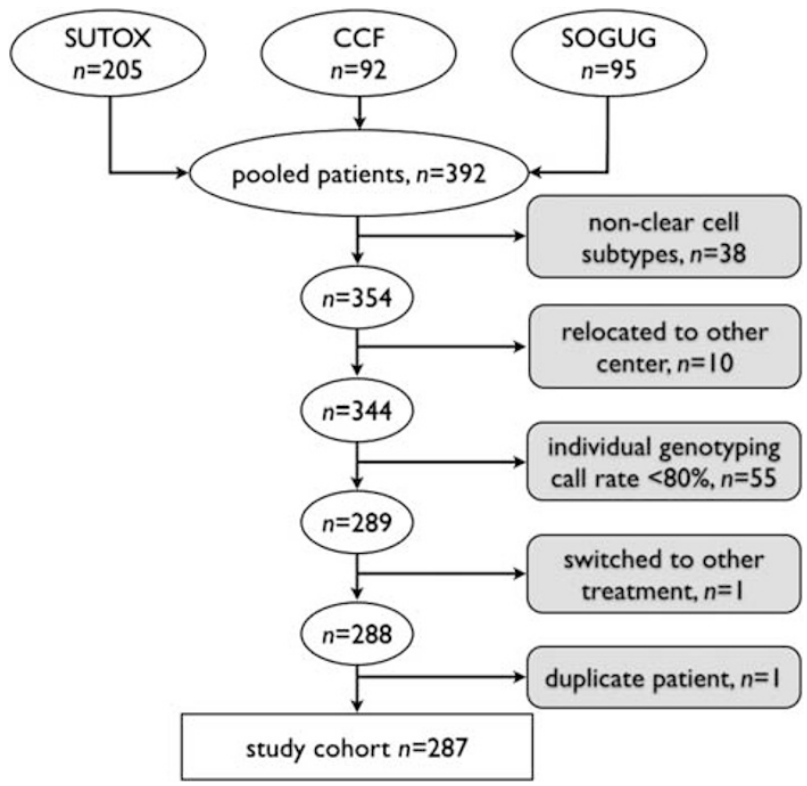

Figure 1. Patient flowchart on included patients. A total of 105 patients had to be excluded from association analyses because of non-clear cell subtypes $(n=38)$, relocation to another medical center during follow-up $(n=10)$, individual genotyping call rates $<80 \%$ $(n=55)$, double patient $(n=1)$ or a change to another treatment than sunitinib directly after enrolment $(n=1)$. A total of 287 sunitinib-treated metastatic renal cell carcinoma patients were available for analysis of toxicity and survival in the present study. CCF, cleveland clinic foundation; SOGUG, Spanish oncology genitourinary group; SUTOX, dutch SUTOX consortium. ${ }^{11}$

working group focusing on genotype-related sunitinib-induced toxicity and comprises five medical centers in the Netherlands. The Spanish Oncology Genitourinary Group (SOGUG) consists of 15 Spanish medical centers, and CCF is the Taussig Cancer Institute of Cleveland Clinic Foundation in the United States. ${ }^{1-5,11}$ SUTOX samples were anonymized by a third party, according to the instructions stated in the Codes for Proper Use and Proper Conduct in the Self-Regulatory Codes of Conduct (www. federa.org). The Medical Ethical review boards of all participating centers approved the study. Patients provided their written informed consent for participation. ${ }^{2,11}$

\section{Genotyping methods}

Germline DNA was isolated from EDTA-whole blood, peripheral blood mononuclear cell samples, serum or plasma. Samples from serum or plasma were preamplified in order to have a higher DNA concentration. DNA samples from SUTOX and CCF patients were genotyped at Leiden University Medical Center using Taqman probes (Applied Biosystems, Nieuwerkerk aan den IJssel, The Netherlands) on the LightCycler480 (LC480) (Roche Applied Science, Almere, The Netherlands) and on ViiA 7500 (Applied Biosystems) Real-Time PCR Instruments. The latter was used for the analysis of preamplified samples for a better fluorescence chart review. DNA samples from SOGUG were genotyped by the Spanish National Cancer Research Centre using a KASPar SNP genotyping system (Kbiosciences, Hoddesdon, UK) and the sequence Detection System 7900HT (Applied Biosystems, Foster City, CA, USA) for fluorescence detection and allele assignment. In all, $5 \%$ of the samples were genotyped in duplicate, and no inconsistencies were observed. ${ }^{11}$

\section{Study design}

Sunitinib toxicity was evaluated during four 6-week treatment cycles and scored at baseline, week 4 and week 6 of each cycle according to the National Cancer Institute-Common Terminology Criteria for Adverse Events (NCI-CTCAE) v3.0 or v4.0. ${ }^{11}$ Tested toxicity outcomes were thrombocytopenia, leukopenia, mucosal inflammation, hand-foot syndrome, hypertension and any toxicity $>$ grade 2 . Toxicities $\geqslant$ grade 3 were considered clinically relevant. Tested toxicity outcomes were adjusted for toxicity grades observed at baseline. ${ }^{11}$ Baseline corrected toxicity scores were calculated by subtracting baseline values from the maximum recorded score in four cycles of treatment. Corrected toxicity scores were divided into grades $0-2$ and grades $3-4 .{ }^{11}$ Patient characteristics considered relevant for suffering any kind of adverse event were age at the start of sunitinib, gender and study center.

For survival analysis, progression-free survival (PFS) was defined as the time in months between the first day of sunitinib and the date of progressive disease (PD) according to the Response Evaluation Criteria in Solid Tumours version 1.0. If a patient had not progressed, PFS was censored at the time of the last follow-up or date of death. Overall survival (OS) was defined as the time in months between the first day of sunitinib and the date of death or the date at which patients were last known to be alive (last follow-up date). Variables considered to affect PFS or OS were age at the start of sunitinib, gender, study center and prognostic risk group according to Heng criteria. ${ }^{11-12}$

\section{Statistical analysis}

Genotyping results were dichotomized in a dominant model with WT (GG genotype) vs A-allele carriers (AG and AA genotype), analogous to the work of Urun et al..$^{6}$ Distribution of genotypes was tested for deviation from Hardy-Weinberg equilibrium using a $x^{2}$ goodness-of-fit test. Distribution of the continuous variable 'age at the start of sunitinib' followed a normal distribution according to the Shapiro-Wilk test.

SNP rs4646437 (WT vs A-allele carriers) was tested univariately against toxicity end points using a $x^{2}$ test. For multivariate analysis, SNP rs 4646437 was tested against toxicity end points dichotomized as grades $0-2$ and grades 3-4 using binary logistic regression $(P<0.05)$, including the covariates age at the start of sunitinib, gender and study center. ${ }^{11}$

For PFS and OS analysis, age, gender, study center and Heng prognostic risk group ${ }^{12}$ together with CYP3A4 rs4646437 genotype were included in the multivariate Cox regression survival analysis. All results with a $P$-value $<0.05$ from multivariate analyses were considered significant.

In addition, the interaction between the SNP rs4646437 genotypes and gender was tested univariately for association with all of the toxicity outcomes and with PFS and OS. ${ }^{10-11}$ This additional analysis was performed to see whether the interaction term of SNP $\times$ gender needed to be included as covariate in multivariate analysis. Interaction between gender and the rs4646437 genotype on PFS and OS was also tested using Cox regression survival analysis.

All tests were two sided and carried out using SPSS Statistical Package for Windows (version 20.0; IBM, Armonk, NY, USA).

\section{RESULTS}

Of the initial 391 patients, 287 clear cell mRCC patients met the inclusion criteria of which $68 \%$ were males (Figure 1). Baseline patient characteristics are summarized in Table 1. Genotype frequencies were 228 carriers of WT GG, 52 carriers of heterozygous AG and 7 carriers of the AA genotype. This is consistent with Hardy-Weinberg equilibrium $\left(x^{2}=3.46, P=0.063\right)$. Obtained minor allele frequency was $6.97 \%$.

Most patients had no toxicities at baseline $(95.5-100 \%$ for the tested toxicities). Baseline grades 1-3 toxicities were observed for $0-4.5 \%$ of patients, and no baseline grade 4 toxicities were observed. Dose reductions were needed for $33 \%$ of patients within cycles 1-4 of sunitinib. Any toxicity > grade 2 was observed in $25 \%$ of patients (Table 2). Observed grades 3-4 adverse events within four cycles of sunitinib were thrombocytopenia (7.7\%) leukopenia (2.8\%), mucosal inflammation (3.1\%), hand-foot syndrome (5.2\%) and hypertension (13.6\%). The interaction between SNP genotype and gender was tested against all toxicity outcomes, but no significant interaction was found in univariate analysis $(P=0.27)$. As a result, gender was included as an independent covariate instead of a combined 'gender $\times$ SNP' covariate. Genotype distributions for males and females are presented in Table 1. Table 3 shows the results of association analyses. For A-allele carriers of CYP3A4 rs4646437, we observed an increased risk for hypertension $(\mathrm{OR}=2.4,95 \% \mathrm{Cl}: 1.1-5.2, P=0.021)$. For other toxicity end points, no significant associations were observed. 
Table 1. Baseline characteristics of clear cell metastatic renal cell carcinoma patients treated with sunitinib $(n=287)$

\begin{tabular}{|c|c|c|}
\hline Feature & Value (range) & $\%$ \\
\hline \multicolumn{3}{|l|}{ Gender } \\
\hline Male & 195 & 68 \\
\hline Female & 92 & 32 \\
\hline Median age at the start of sunitinib, years & $61(34-87)$ & \\
\hline Mean BSA & $1.97(1.2-2.7)$ & \\
\hline \multicolumn{3}{|l|}{ Prior nephrectomy } \\
\hline Yes & 242 & 84.3 \\
\hline No & 41 & 14.3 \\
\hline Unknown & 4 & 1.4 \\
\hline \multicolumn{3}{|l|}{ WHO performance status } \\
\hline 0 & 120 & 42 \\
\hline 1 & 142 & 50 \\
\hline 2 & 17 & 6 \\
\hline 3 & 1 & 0 \\
\hline Unknown & 7 & 2 \\
\hline \multicolumn{3}{|l|}{ Ethnicity } \\
\hline Caucasian & 277 & 97 \\
\hline Black & 4 & 1 \\
\hline Asian & 2 & 1 \\
\hline Arab & 3 & 1 \\
\hline Latin American & 1 & 0 \\
\hline \multicolumn{3}{|l|}{ Gender genotype distributions } \\
\hline Female AA carriers & 2 & 1 \\
\hline Female AG carriers & 17 & 6 \\
\hline Female GG carriers & 73 & 25 \\
\hline Male AA carriers & 5 & 2 \\
\hline Male AG carriers & 35 & 12 \\
\hline Male GG carriers & 155 & 54 \\
\hline \multicolumn{3}{|l|}{ Heng prognostic risk group ${ }^{\mathrm{a}}$} \\
\hline Good (0 risk factors) & 61 & 21 \\
\hline Intermediate (1-2 risk factors) & 157 & 55 \\
\hline Poor (3-6 risk factors) & 69 & 24 \\
\hline \multicolumn{3}{|l|}{ Prior systemic antitumor treatment } \\
\hline Yes & 52 & 18 \\
\hline No & 235 & 82 \\
\hline \multicolumn{3}{|l|}{ Sunitinib daily dose (mg) } \\
\hline Only 50 & 269 & 94 \\
\hline Only 37.5 & 14 & 5 \\
\hline Only 25 & 4 & 1 \\
\hline \multicolumn{3}{|l|}{ Dose reduction after cycle 1, 2 or 3} \\
\hline No & 180 & 63 \\
\hline Yes & 95 & 33 \\
\hline Unknown & 12 & 4 \\
\hline \multicolumn{3}{|l|}{ Best response to sunitinib } \\
\hline Complete response & 9 & 3 \\
\hline Partial response & 120 & 42 \\
\hline Stable disease & 104 & 36 \\
\hline Progressive disease & 37 & 13 \\
\hline Unknown & 17 & 6 \\
\hline Median SBP (mm Hg) & $140(95-205)$ & \\
\hline Median DBP (mm Hg) & $80(29-110)$ & \\
\hline Median LDH $\left(\mathrm{U} \mathrm{I}^{-1}\right)$ & $241(110-1190)$ & \\
\hline Median creatinine $\left(\mu \mathrm{mol} \mathrm{I}{ }^{-1}\right)$ & $106.1(1.0-247.5)$ & \\
\hline Median bilirubin $\left(\mu \mathrm{mol} \mathrm{I}^{-1}\right)$ & $6.84(0.5-46.2)$ & \\
\hline Median albumin $\left(\mathrm{g} \mathrm{I}^{-1}\right)$ & $42(4.7-51.0)$ & \\
\hline Median AST $\left(\mathrm{UI}^{-1}\right)$ & $19(8-107)$ & \\
\hline Median ALT $\left(\mathrm{UI}^{-1}\right)$ & $17(4-119)$ & \\
\hline Median hemoglobin $\left(\mathrm{mmol} \mathrm{I}^{-1}\right)$ & $8.1(4.7-14.2)$ & \\
\hline
\end{tabular}

The median PFS was 17 months with a median follow-up period of 45 months for PFS analysis. The median follow-up period was 49 months for OS and the median OS was 29 months. The interaction between gender and genotype was tested in PFS and OS analysis, but no significant interaction was found $(P=0.23$ and $P=0.76$ respectively). There was no significant difference in median PFS or median OS between CYP3A4 rs4646437 WT and A-allele carrier patients both uncorrected and corrected for age, gender, study center and Heng prognostic risk group in Cox regression survival analysis (Table 3 ).

\section{DISCUSSION}

In this study, we observed a significant association between the presence of A-allele in CYP3A4 rs4646437 and an increased risk for hypertension compared with WT carriers in sunitinib-treated mRCC patients. Hence, CYP3A4 rs4646437 is a potential predictive biomarker for hypertension in sunitinib-treated mRCC patients, but this needs confirmation.

This is the first time that SNP CYP3A4 rs4646437 is examined in a large cohort of mRCC patients. Grades 3-4 hypertension was observed in $14 \%$ of patients both in this study and in the study of Urun et al. ${ }^{6}$ Our findings do not confirm the data of Urun et al., ${ }^{6}$ who observed no association with hypertension and who reported a reduced risk of grades 3-4 toxicities for AG carriers of CYP3A4 rs4646437 (OR $=0.27)$. In contrast to earlier reports, we did not demonstrate significant results for the interaction between genotype and gender. ${ }^{10}$

Our studied time period of four treatment cycles in which toxicity data were collected is comparable to Urun et $a l^{6}{ }^{6}$ who reported a median treatment duration of 7.7 months. In both studies, Caucasians were included of whom the majority were male. $^{6}$ The difference in our findings with those of the group of Urun et al. ${ }^{6}$ could partly be explained by our large sample size (287 vs 159 patients), a higher number of A carriers (20.6\% vs $11.2 \%)$ and a lower rate of adverse events > grade $2(24.7 \%$ vs $52 \%)$. In addition, our fixed covariates in multivariate testing $(P<0.1)$ differ from the included clinical covariates by Urun et al. ${ }^{6}$ using a significance threshold of $P<0.2$. The time frame of our study is long including 7 years, which could introduce a bias as clinical experience with sunitinib may have caused dose reduction before the occurrence of $>$ grade 2 adverse events. A time-toevent analysis is preferred for toxicity analysis as many patients will not complete four cycles of sunitinib treatment, and patients may develop toxicities earlier or later in this time frame. However, it was not possible to collect these data accurately because of the retrospective character of this study.

\begin{tabular}{lcc}
\hline Table 1. (Continued) & \\
\hline Feature & Value (range) & $\%$ \\
\hline Median leukocytes $\left(\times 10^{9}\right)$ & $7.6(1.6-50.0)$ \\
Median neutrophils $\left(\times 10^{9}\right)$ & $4.8(0.7-26.4)$ \\
Median thrombocytes $\left(\times 10^{9}\right)$ & $254(92-1492)$ \\
Median MCV $(\mathrm{fl})$ & $88(68-110)$ \\
Median calcium $\left(\mathrm{mmol} \mathrm{I}^{-1}\right)$ & $2.38(1.92-2.93)$ \\
\hline
\end{tabular}

Abbreviations: ALT, alanine transaminase; AST, aspartate transaminase; BSA, body surface area; DBP, diastolic blood pressure; LDH, lactate dehydrogenase; MCV, mean corpuscular volume; SBP, systolic blood pressure; WHO, world health organization (performance status). ${ }^{\text {aP }}$ Patients are grouped according to their Heng risk group based on the six Heng risk scores: detoriated WHO performance status $(\geqslant 2)$, low hemogblobin $(<$ lower limit of normal), high calcium $\left(>2.5 \mathrm{mmolI}^{-1}\right)$, time from initial diagnosis to treatment with sunitinib ( $<1$ year), neutrophil count (greater than upper limit of normal) and thrombocytes (greater than upper limit of normal). ${ }^{11}$ 


\begin{tabular}{|c|c|c|c|}
\hline Type of toxicity & $\begin{array}{l}\text { Toxicity } \\
\text { grade }\end{array}$ & $\begin{array}{l}\text { Number of } \\
\text { patients (n) }\end{array}$ & $\begin{array}{l}\text { Percentage of } \\
\text { patients (\%) }\end{array}$ \\
\hline \multirow[t]{5}{*}{ Thrombocytopenia } & None & 104 & 36 \\
\hline & Grade 1 & 122 & 43 \\
\hline & Grade 2 & 39 & 14 \\
\hline & Grade 3 & 18 & 6 \\
\hline & Grade 4 & 4 & 1 \\
\hline \multirow[t]{4}{*}{ Leukopenia } & None & 151 & 53 \\
\hline & Grade 1 & 78 & 27 \\
\hline & Grade 2 & 50 & 17 \\
\hline & Grade 3 & 8 & 3 \\
\hline \multirow[t]{4}{*}{ Mucosal inflammation } & None & 126 & 44 \\
\hline & Grade 1 & 93 & 32 \\
\hline & Grade 2 & 59 & 21 \\
\hline & Grade 3 & 9 & 3 \\
\hline \multirow[t]{4}{*}{ Hand-foot syndrome ${ }^{a}$} & None & 173 & 60 \\
\hline & Grade 1 & 57 & 20 \\
\hline & Grade 2 & 42 & 15 \\
\hline & Grade 3 & 15 & 5 \\
\hline \multirow[t]{5}{*}{ Any toxicity $>$ grade 2} & None & 39 & 14 \\
\hline & Grade 1 & 76 & 26 \\
\hline & Grade 2 & 101 & 35 \\
\hline & Grade 3 & 62 & 22 \\
\hline & Grade 4 & 9 & 3 \\
\hline \multirow[t]{4}{*}{ Hypertension } & None & 173 & 60 \\
\hline & Grade 1 & 29 & 10 \\
\hline & Grade 2 & 45 & 16 \\
\hline & Grade 3 & 39 & 14 \\
\hline \multicolumn{4}{|c|}{$\begin{array}{l}\text { In case the grade of toxicity was not recorded in the medical record of the } \\
\text { patient, it was assumed that no toxicity had occurred (grade } 0 \text { ). }{ }^{\text {a For hand- }} \text { - } \\
\text { foot syndrome, grade } 3 \text { is the highest possible grade according to CTCAE } \\
\text { version } 4.0 \text {. }\end{array}$} \\
\hline
\end{tabular}

The expression of CYP3A4 in the liver is twofold higher in women than in men that can be explained by sex-dependent gene expression differences. ${ }^{10}$ However, as genders are not equally present in this study (only one-third is female) and merely 19 subjects $(7 \%)$ were female A carriers, it is not possible to perform robust statistics for interaction testing between gender and SNP genotype.

Based on our results in this large cohort, it remains difficult to explain whether A-allele carriers of CYP3A4 rs4646437 either have an increased or decreased function of CYP3A4. One could reason that an increased function would lead to conversion of sunitinib to the metabolite SU12662 with a similar activity and a longer halflife compared with the parent compound. On the other hand, a decreased function of CYP3A4 could lead to an increase in total drug exposure all the same. In both hypotheses, A-allele carriers will have an increased exposure to the drug with a stronger inhibition of vascular endothelial growth factor receptor 2 (VEGFR-2) and therefore a higher risk of hypertension. ${ }^{2-4,13}$

A number of PK/pharmacodynamic analyses demonstrate that the increased plasma drug exposure of sunitinib (and SU12662) indeed results in hypertension or other types of toxicity. In a large meta-analysis of Houk et al. ${ }^{14}$ on mainly Caucasian patients, it was shown that a higher exposure to sunitinib and SU12662 (total drug AUC) resulted in improved clinical outcomes and an increased risk of adverse events: fatigue, diastolic blood pressure elevation, or reduction of the absolute neutrophil count. Also, in a PK analysis of Mizuno et al. ${ }^{15}$ on 19 Japanese RCC patients, the systemic exposure to sunitinib and its active metabolite was associated with the incidence of thrombocytopenia ( $\geqslant$ grade 2) and hypertension ( $\geqslant$ grade 2 ). Nagata et al. ${ }^{16}$ studied six Japanese RCC patients of whom four received a starting dose of $50 \mathrm{mg}$ and
Table 3. Association analysis results of CYP3A4 rs4646437 vs toxicity end points and survival end points

\begin{tabular}{|c|c|c|c|c|}
\hline \multirow[t]{2}{*}{ Toxicity end point } & \multirow[t]{2}{*}{ P-value } & \multirow[t]{2}{*}{ OR } & \multicolumn{2}{|c|}{$95 \% \mathrm{Cl}$} \\
\hline & & & Lower & Upper \\
\hline \multicolumn{5}{|c|}{ Thrombocytopenia grades $0-2$ vs $3-4$} \\
\hline WT vs A carriers uncorrected & 0.18 & 0.31 & 0.08 & 1.61 \\
\hline WT vs A carriers & 0.21 & 0.38 & 0.08 & 1.74 \\
\hline \multicolumn{5}{|l|}{ Leukopenia grades $0-2$ vs 3-4 } \\
\hline WT vs A carriers uncorrected & 0.57 & 0.54 & 0.07 & 4.5 \\
\hline WT vs A carriers & 0.78 & 0.73 & 0.08 & 6.4 \\
\hline \multicolumn{5}{|l|}{ Mucositis grades $0-2$ vs $3-4$} \\
\hline WT vs A carriers uncorrected & 0.90 & 1.11 & 0.22 & 5.48 \\
\hline WT vs A carriers & 0.72 & 1.37 & 0.25 & 7.5 \\
\hline \multicolumn{5}{|c|}{ Hand-foot syndrome grades $0-2$ vs $3-4^{a}$} \\
\hline WT vs A carriers uncorrected & 0.07 & 2.76 & 0.94 & 8.08 \\
\hline WT vs A carriers & 0.14 & 2.33 & 0.77 & 7.09 \\
\hline \multicolumn{5}{|l|}{ Hypertension grades $0-2$ vs $3-4$} \\
\hline WT vs A carriers uncorrected & 0.013 & 2.51 & 1.21 & 5.22 \\
\hline Wt vs A carriers & 0.021 & 2.43 & 1.14 & 5.18 \\
\hline \multicolumn{5}{|l|}{ Any toxicity $>$ grade 2} \\
\hline WT vs A carriers uncorrected & 0.59 & 0.83 & 0.42 & 1.64 \\
\hline WT vs A carriers & 0.89 & 0.95 & 0.46 & 1.96 \\
\hline \multirow[t]{2}{*}{ Survival end point } & P-value & $H R$ & \multicolumn{2}{|c|}{$95 \% \mathrm{Cl}$} \\
\hline & & & Lower & Upper \\
\hline \multicolumn{5}{|l|}{ PFS } \\
\hline WT vs A carriers uncorrected & 0.38 & 0.86 & 0.61 & 1.20 \\
\hline WT vs A carriers & 0.14 & 0.77 & 0.55 & 1.09 \\
\hline \multicolumn{5}{|l|}{ OS } \\
\hline WT vs $A$ carriers uncorrected & 0.92 & 1.02 & 0.71 & 1.47 \\
\hline WT vs $A$ carriers & 0.45 & 0.86 & 0.59 & 1.26 \\
\hline
\end{tabular}

Abbreviations: $\mathrm{Cl}$, confidence interval; $\mathrm{HR}$, hazard ratio; $\mathrm{OR}$, odds ratio; $\mathrm{OS}$, overall survival; PFS, progression-free survival; WT, wild type. In case the grade of toxicity was not recorded in the medical record of the patient, it was assumed that no toxicity had occurred (grade 0 ). Toxicity end points are corrected for age, gender and study center. Survival end points are corrected for age, gender, Heng prognostic risk group and study center. ${ }^{\text {a}}$ For hand-foot syndrome, grade 3 is the highest possible grade according to CTCAE version 4.0. Values in bold represent $P$-values $<0.05$ corrected for clinical covariates.

developed grade 2 or 3 thrombocytopenia. It was concluded that the total trough level of sunitinib should be $<100 \mathrm{ng} \mathrm{ml}^{-1}$ in order to avoid severe thrombocytopenia and the starting dose could be reduced to 37.5 or $25 \mathrm{mg}$ in most cases. In another PK analysis, Noda et al. ${ }^{17}$ investigated 21 RCC patients on the association of total sunitinib concentration with toxicities and clinical outcome. Patients with a total sunitinib concentration $\geqslant 100 \mathrm{ng} \mathrm{ml}^{-1}$ showed a higher incidence of $\geqslant$ grade 3 thrombocytopenia, anorexia or fatigue. ${ }^{17}$ Recently, Teo et al. ${ }^{18}$ investigated the effect of SNPs in CYP3A5 and $A B C B 1$ on both PK and clinical outcome (that is, toxicity and response) in a Chinese population of 31 patients. The variant CC genotype of rs 1045642 in $A B C B 1$ was associated with a higher exposure to sunitinib, an increased risk for rash and mucositis and progression of the disease as compared with T-allele carriers $(P<0.05)$. No association with sunitinib exposure, response or toxicities was seen for rs776746 in CYP3A5. ${ }^{18}$ In contrast, our research group investigated 
a considerably larger group of Caucasian patients and reported that rs776746 in CYP3A5 was associated with hypertension $(\mathrm{OR}=4.70,95 \% \mathrm{Cl}: 1.47-15.0, P=0.009)$ and with the need for dose reductions. ${ }^{11}$ Another SNP in CYP3A4 (CYP3A4*22) was tested in a set of 114 patients and associated with a $22.5 \%$ decrease in clearance of sunitinib, which explains the higher exposure to the drug. ${ }^{19}$ However, this pharmacogenetic-PK analysis did not investigate the occurrence of toxicities and the SNP is different from that in the present study. In putting together these results, we should be aware that patients of Asian ethnicity have been reported to have a higher incidence of severe sunitinib-induced toxicities compared with Caucasians. ${ }^{11,14-18}$

Remarkable in our findings is that only an association was observed of SNP rs4646437 with sunitinib-induced hypertension, whereas no associations have been found with any of the other tested toxicities on sunitinib. Although mechanisms are currently not fully unraveled, it is thought that sunitinib-induced hypertension is caused by inhibition of VEGFR-2 leading to a reduced amount of nitric oxide and thus vasoconstriction. SNPs in VEGFA and eNOS are reported to be related to sunitinib-induced hypertension. ${ }^{4,11,13}$ Probably there are more factors involved that influence the development of hypertension, which can be related to both PK and pharmacodynamics. The current study and other recent findings of our group suggest that SNPs in CYP3A4 and CYP3A5 could have an essential role in hypertension development. ${ }^{11}$

Furthermore, hypertension can be considered as a different sort of toxicity as it is related to improved clinical outcomes on sunitinib. ${ }^{4,20}$ Yet, no improved survival outcomes were seen for the variant A-allele carriers of rs4646437. Promising biomarkers for sunitinib efficacy have been identified in $A B C B 1$. Corresponding results were observed for SNPs in $A B C B 1$ : the presence of WT CGT haplotype in rs1128503, rs2032582 and rs1045642 resulted in an improved clinical outcome (PFS, OS and response), the CC WT in rs1128503 showed a decrease in sunitinib clearance, and the $\Pi$ variant in rs1128503 required less dose reductions. ${ }^{11,19,21}$ In contrast, the variant CC genotype of rs1045642 showed an increased exposure to sunitinib in Japanese patients. ${ }^{18}$ To predict hypertension and subsequent effects on survival, we may be searching for one biomarker that consists of multiple SNPs related to the PK and pharmacodynamics of sunitinib.

In conclusion, hypertension is more likely to occur in A-allele carriers of the CYP3A4 rs4646437 variant. Confirmation of this finding in another cohort is needed to potentially use this SNP as a predictive biomarker for hypertension on sunitinib treatment.

\section{CONFLICT OF INTEREST}

Brian Rini and Garcia-Donas report consulting and research funding from Pfizer. The other authors declare no conflict of interest.

\section{ACKNOWLEDGMENTS}

We thank Tahar van der Straaten and Renée Baak-Pablo for their assistance with data management and genotyping. The European Union's Seventh Framework Programme (FP7/2007-2013) supports Meta Diekstra under grant agreement no. 259939. This research is supported by Pfizer.

\section{REFERENCES}

1 van der Veldt A, Eechoute K, Gelderblom H, Gietema J, Guchelaar H-J, van Erp N et al. Genetic polymorphisms associated with a prolonged progression-free survival in patients with metastatic renal cell cancer treated with sunitinib. Clin Cancer Res 2011; 17: 620-629.

2 van Erp N, Eechoute K, van der Veldt A, Haanen J, Reyners A, Mathijssen R et al. Pharmacogenetic pathway analysis for determination of sunitinib-induced toxicity. J Clin Oncol 2009; 27: 4406-4412.
3 Garcia-Donas J, Esteban E, Leandro-Garcia L, Castellano D, del Alba A, Climent M et al. Single nucleotide polymorphism associations with response and toxic effects in patients with advanced renal-cell carcinoma treated with first-line sunitinib: a multicentre, observational, prospective study. Lancet Oncol 2011; 12: 1143-1150.

4 Eechoute K, van der Veldt A, Oosting S, Kappers M, Wessels J, Gelderblom H et al. Polymorphisms in endothelial nitric oxide synthase (eNOS) and vascular endothelial growth factor (VEGF) predict sunitinib-induced hypertension. Clin Pharmacol Ther 2012; 92: 503-510.

5 Kim J, Vaziri S, Rini B, Elson P, Garcia J, Wirka R et al. Association of VEGF and VEGFR2 single nucleotide polymorphisms with hypertension and clinical outcome in metastatic clear cell renal cell carcinoma patients treated with sunitinib. Cancer 2012; 118: 1946-1954.

6 Urun Y, Gray K, Signoretti S, McDermott D, Atkins M, Lampron M et al. Pharmacogenetics as predictor of sunitinib and mTOR inhibitors toxicity in patients with metastatic renal cell carcinoma (mRCC). ASCO Meeting Abstr 2013; 31; http://meetinglibrary.asco.org/content/113157-132.

$7 \mathrm{He} \mathrm{H}$, Sun J, Ren X, Wang T, Zhai Y, Chen S et al. Effects of CYP3A4 polymorphisms on the plasma concentration of voriconazole. Eur J Clin Microbiol Infect Dis 2015; 34: 811-819.

8 Li C, Li L, Lin L, Jiang H, Zhong Z, Li W et al. Impact of the CYP3A5, CYP3A4, COMT, IL-10 and POR genetic polymorphisms on tacrolimus metabolism in Chinese renal transplant recipients. PLoS One 2014; 9: e86206.

9 Crettol S, Venetz J-P, Fontana M, Aubert J-D, Pascual M, Eap C et al. CYP3A7, CYP3A5, CYP3A4, and ABCB1 genetic polymorphisms, cyclosporine concentration, and dose requirement in transplant recipients. Ther Drug Monit 2008; 30: 689-699.

10 Schirmer M, Rosenberger A, Klein K, Kulle B, Toliat M, Nurnberg P et al. Sexdependent genetic markers of CYP3A4 expression and activity in human liver microsomes. Pharmacogenomics J 2007; 8: 443-453.

11 Diekstra M, Swen J, Boven E, Castellano D, Gelderblom H, Mathijssen R et al. CYP3A5 and $A B C B 1$ polymorphisms as predictors for sunitinib outcome in metastatic renal cell carcinoma. Eur Urol 2015; 68: 621-629.

12 Heng D, Xie W, Regan M, Warren M, Golshayan A, Sahi C et al. Prognostic factors for overall survival in patients with metastatic renal cell carcinoma treated with vascular endothelial growth factor-targeted agents: results from a large, multicenter study. J Clin Oncol 2009; 27: 5794-5799.

13 Lankhorst S, Kappers M, van Esch J, Danser A, van den Meiracker A. Hypertension during vascular endothelial growth factor inhibition: focus on nitric oxide, endothelin-1, and oxidative stress. Antioxid Redox Signal 2014; 20: 135-145.

14 Houk B, Bello C, Poland B, Rosen L, Demetri G, Motzer R et al. Relationship between exposure to sunitinib and efficacy and tolerability endpoints in patients with cancer: results of a pharmacokinetic/pharmacodynamic meta-analysis. Cancer Chemother Pharmacol 2010; 66: 357-371.

15 Mizuno T, Fukudo M, Terada T, Kamba T, Nakamura E, Ogawa O et al. Impact of genetic variation in breast cancer resistance protein (BCRP/ABCG2) on sunitinib pharmacokinetics. Drug Metab Pharmacokinet 2012; 27: 631-639.

16 Nagata M, Ishiwata Y, Takahashi Y, Takahashi H, Saito K, Fujii $Y$ et al. Pharmacokinetic-pharmacodynamic analysis of sunitinib-induced thrombocytopenia in Japanese patients with renal cell carcinoma. Biol Pharm Bull 2015; 38: 402-410.

17 Noda S, Otsuji T, Baba M, Yoshida T, Kageyama S, Okamoto K et al. Assessment of sunitinib-induced toxicities and clinical outcomes based on therapeutic drug monitoring of sunitinib for patients with renal cell carcinoma. Clin Genitourin Cancer 2015; 13: 350-358.

18 Teo Y, Wee H, Chue X, Chau N, Tan M, Kanesvaran R et al. Effect of the CYP3A5 and $A B C B 1$ genotype on exposure, clinical response and manifestation of toxicities from sunitinib in Asian patients. Pharmacogenomics J 2015; e-pub ahead of print 17 March 2015; doi:10.1038/tpj.2015.13

19 Diekstra M, Klümpen HJ, Lolkema M, Yu H, Kloth J, Gelderblom H et al. Association analysis of genetic polymorphisms in genes related to sunitinib pharmacokinetics, specifically clearance of sunitinib and SU12662. Clin Pharmacol Ther 2014; 96: 81-89.

20 Rini B, Cohen D, Lu D, Chen I, Hariharan S, Gore M et al. Hypertension as a biomarker of efficacy in patients with metastatic renal cell carcinoma treated with sunitinib. J Nat/ Cancer Inst 2011; 103: 763-773.

21 Beuselinck B, Lambrechts D, Van Brussel T, Wolter P, Cardinaels N, Joniau S et al. Efflux pump $A B C B 1$ single nucleotide polymorphisms and dose reductions in patients with metastatic renal cell carcinoma treated with sunitinib. Acta Oncol 2014; 53: 1413-1422. 